\title{
Derivative-order-dependent stability and transient behaviour in a predator-prey system of fractional differential equations
}

\author{
Z. M. Alqahtani (1Da ${ }^{a}$ M. El-Shahed $\mathbb{1}^{\mathrm{b}}$ and N. J. Mottram (1) ${ }^{\mathrm{C}}$ \\ a Department of Mathematical Sciences, Faculty of Sciences, Princess Nourah Bint Abdulrahman University,

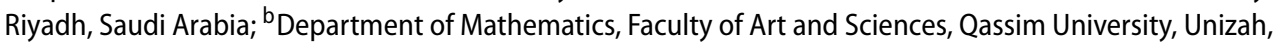 \\ Saudi Arabia; ' Department of Mathematics \& Statistics, University of Strathclyde, Glasgow, UK
}

\begin{abstract}
In this paper, the static and dynamic behaviour of a fractional-order predator-prey model are studied, where the nonlinear interactions between the two species lead to multiple stable states. As has been found in many previous systems, the stability of such states can be dependent on the fractional order of the time derivative, which is included as a phenomenological model of memory-effects in the predator and prey species. However, what is less well understood is the transient behaviour and dependence of the observed domains of attraction for each stable state on the order of the fractional time derivative. These dependencies are investigated using analytical (for the stability of equilibria) and numerical (for the observed domains of attraction) techniques. Results reveal far richer dynamics compared to the integer-order model. We conclude that, as well as the species and controllable parameters, the memory effect of the species will play a role in the observed behaviour of the system.
\end{abstract}

\section{ARTICLE HISTORY}

Received 19 November 2018

Accepted 8 August 2019

\section{KEYWORDS}

Population dynamics; mathematical modelling; fractional calculus; domain of attraction

\section{Introduction}

The study of fractional calculus has a long history, dating back to work by Leibniz and L'Hospital, when half-order derivatives were considered (Kilbas, Srivastava, \& Trujillo, 2006). Since then many authors have proposed forms of fractional derivatives, the most commonly used being the Riemann-Liouville fractional derivative and the Caputo fractional derivative (Podlubny, 1999). In recent years differential equations which involve fractional derivatives have been studied by many authors as models of numerous systems in different branches of physics, chemistry and biological sciences (see, for instance, Heymans \& Podlubny, 2006). This generalization of integer-order systems to include fractional derivatives has often been motivated by a desire to describe complex systems with nonlocal interactions in time and space (Miller \& Ross, 1993; Oldham \& Spanier, 1974). In these models, the Caputo fractional derivative is often preferred since it has the advantage over the Riemann-Liouville form that initial conditions need only contain integer-order derivatives rather than fractional-order derivatives. In fact, the Caputo derivative was first

CONTACT Z.M.Alqahtani zhr_math@hotmail.com 
developed as a model of memory effects in material science, with dissipation of a viscoelastic material being written as a weighted integral of the first-order derivative of strain and a memory kernel (Caputo, 1967; Caputo \& Mainardi, 1971). For these reasons we will also use the Caputo definition of a fractional derivative throughout this paper.

In the last few decades, mathematical models of population dynamics have attracted wide attention and, in particular, predator-prey systems have been extensively studied and used to explain the stable and periodic dynamics of interacting species (Murray, 2004). For such systems, the Lotka-Volterra model of predator-prey systems has been widely used, and extended, to enable relatively simple and more complicated food-web interactions (Freedman, 1980). The more complicated predator-prey models include refinements in the modelling of growth, death, predation, harvesting and multiple species (Chattopadhyay, Sarkar, \& Ghosal, 2002; Dai \& Tang, 1998; Fenton \& Perkins, 2010; Freedman \& Waltman, 1984; Rahman \& Chakravarty, 2013; Srinivasu, Ismail, \& Naidu, 2001). In such models of biological interaction the rate of change of dependent variables, such as populations and biomass, have been calculated as time derivatives of order one, and depend only on the dependent variables evaluated at the present time. However, for some biological phenomena, often termed memory or delayed effects, changes in the current system state depend on states in past times. In some situations, such as a conception-gestationbirth process, memory effects need only to reflect a dependence on a state at a fixed time in the past, and therefore a delay-differential equation can be used successfully (Forde, 2005; Kuang, 1993; Milton, 2015). If there are a number of identifiable delayed responses in the system (i.e. conception-gestation, disease incubation periods, birth-to-maturity times, and for many species in the model) then a system of delay-differential equations with multiple delays can be developed (Freedman \& Rao, 1986). However, when memory is distributed across a continuum of previous times an alternative approach must be considered. Caputo originally developed a specific form of fractional derivative to consider the memory effects in materials (Caputo, 1967; Caputo \& Mainardi, 1971), where the present system state had memory of all previous states, with a weighting governed by a 'memory kernel', and which resulted in the need to specify a convolution of the memory kernel with state derivatives. For mathematical simplicity an algebraically decaying kernel was chosen. Since then the Caputo derivative has been used to model memory in many areas of physics (Richard, 2014) such as viscoelastic fluids (Caputo \& Mainardi, 1971), diffusion close to and in a porous biomembrane (Caputo \& Cametti, 2008, 2009), sedimentation in water reservoirs (Caputo \& Carcione, 2013), plastic media (Caputo, 2016), tumour growth (Arshad, Baleanu, Huang, Tang, \& Al Qurashi, 2016; Bolton, Cloot, Schoombie, \& Slabbert, 2014) as well as in fractional derivative-order population models (ElShahed \& Alsaedi, 2011; Li, Chen, \& Podlubny, 2009; Pooseh, Rodrigues, \& Torres, 2011; Rihan, 2013; Zhang, Chen, Li, \& Kurths, 2013), epidemiology (Pinto \& Carvalho, 2017) and human memory (Du, Wang, \& Hu, 2013). In all of these applications, the introduction of a fractional derivative to model memory is somewhat phenomenological, in that it is understood that some dependence on previous states is needed to introduce memory effects, but that without a particular model of how memory incorporates the information from past times the memory kernel used is chosen for algebraic simplicity and for the fact that it is characterized by a single parameter, the fractional derivative order $\alpha$. While this approach means that the parameter $\alpha$ can only be determined through fitting empirical data, a number of authors have found that such a fitting process can provide a 
better comparison to experimental data than alternative, integer-order derivative models (see, for instance, Bolton et al., 2014; Caputo, 2018; Caputo \& Cametti, 2008, 2009; Caputo $\&$ Carcione, 2013; Du et al., 2013). In the present work we aim to model memory in a phenomenological way and, because there is no specific value of time delay, we use a fractional derivative approach to model memory as an effect distributed in time.

In the works mentioned above, researchers have investigated the stability, existence, uniqueness and numerical solution of fractional derivative models and found that variations in the fractional derivative order affects stability but not existence of equilibrium solutions. In this paper, we consider a fractional derivative generalization of a relatively standard model of population dynamics and investigate the observed domains of attraction for the various stable equilibrium points as a function of the fractional order of the derivatives. By the term observed domains of attraction we do not consider the true asymptotic domains of attraction, i.e. the sets of initial conditions for which the system achieves each of the equilibrium states at infinite time, but rather we classify the initial conditions as being associated with the equilibrium state it is closest to (using the Euclidean distance in state-space) after a fixed observation time. It is these domains that we investigate while varying the fractional order of the time derivatives. Even though the model of memory is phenomenological, the aim in this paper is to show that the fractional derivative order can play a significant role in determining the size of the domain of attraction, and thus has potential to affect real-life observation of the dynamics of interacting populations. We speculate that changes in fractional order, that may occur when memory functions change in time or through environmental effects, have the potential to alter the long time dynamics of a system, particularly when there are two possible stable states.

In the next section, we introduce the fractional-order differential equations to be considered as a model of a two-species predator-prey system. In Sections 3 and 4, the equilibrium states and the stability of these states are investigated, and in Section 5 numerical solutions of the system, found using an Adams-Bashforth-Moulton predictor-corrector scheme, are presented in order to show the effect of fractional order on the dynamics. Finally, conclusions are provided in Section 6.

\section{Predator-prey model}

In this paper, we take, as an example system, the two-species predator-prey model of Kar, Chakraborty, and Pahari (2010),

$$
\begin{aligned}
& \frac{\mathrm{d} x}{\mathrm{~d} t}=r_{1} x\left(1-\frac{x}{k_{1}}\right)-\frac{m x y}{1+a x}-h_{1} x, \\
& \frac{\mathrm{d} y}{\mathrm{~d} t}=r_{2} y\left(1-\frac{y}{k_{2}}\right)+q \frac{m x y}{1+a x}-h_{2} y,
\end{aligned}
$$

where $x(t)$ and $y(t)$ are the prey and predator populations at time $t$, respectively, and we take initial conditions $x(0)=x_{0}$ and $y(0)=y_{0}$. In these equations, the first terms on the right-hand sides of both equations represent the logistic growth of the species with $r_{1}$ and $r_{2}$ being the intrinsic growth rates and $k_{1}$ and $k_{2}$ are the respective environmental carrying capacities of the prey and predator populations, respectively. The second terms on the right-hand sides of (1) and (2) represent the predation of the prey by the predator through 
a Holling type II functional response (Dawes \& Souza, 2013), where $m$ is the percentage decrease of prey per time per predator, which is effectively the efficiency of the predator's ability to capture prey, $a$ is the Michaelis-Menten constant which is related to the predator's efficiency in capture and consumption of the prey, and $q$ is an efficiency constant, which lies between 0 and 1 and which represents the proportion of the prey biomass that the predator can utilize for growth. The last terms in (1) and (2), $h_{1}$ and $h_{2}$, represent the harvesting rate of the prey and predator population, respectively, although it is possible for these terms to be incorporated into adjusted birth rates and carrying capacities if necessary.

For mathematical simplicity, and to reduce the number of independent parameters in the system, we first nondimensionalize (1) and (2) using the nondimensionalized independent variables

$$
\bar{t}=r_{1} t, \quad X=\frac{x}{k_{1}}, \quad Y=\frac{y}{k_{2}},
$$

so that (1) and (2) take the form

$$
\begin{aligned}
& \frac{\mathrm{d} X}{\mathrm{~d} \bar{t}}=X(1-X)-\frac{K_{1} X Y}{1+I X}-E_{1} X, \\
& \frac{\mathrm{d} Y}{\mathrm{~d} \bar{t}}=R Y(1-Y)+\frac{K_{2} X Y}{1+I X}-E_{2} Y,
\end{aligned}
$$

where

$$
\begin{aligned}
E_{1} & =\frac{h_{1}}{r_{1}}, \quad E_{2}=\frac{h_{2}}{r_{1}}, \quad I=a k_{1}, \\
R & =\frac{r_{2}}{r_{1}}, \quad K_{1}=\frac{k_{2} m}{r_{1}}, \quad K_{2}=\frac{k_{1} m q}{r_{1}}
\end{aligned}
$$

are positive nondimensional constants. The constants $E_{1}$ and $E_{2}$ are the ratios of harvesting to birth rate for the prey and predators which, as we will see later, are important to the stability of the populations of each species. The constant $R$ is a ratio of the birth rates of the two species and, to a large extent, is responsible for determining the ratio of predator and prey population numbers at equilibrium. The two parameters $K_{1}$ and $K_{2}$ can be thought of as nondimensionalized carrying capacities for the two species, scaled by the ratio of birth rate and predation factors. The final parameter $I$ is a rescaled, and nondimensional, Michaelis-Menten constant, which measures the efficiency of the predator's search and feeding process.

The aim of this paper is to study the dynamical properties of a generalization of the system described in (3) and (4) through the introduction of fractional-order derivatives. We write this generalized system as

$$
\begin{aligned}
& { }^{c} D_{t}^{\alpha} X=X(1-X)-\frac{K_{1} X Y}{1+I X}-E_{1} X, \\
& { }^{c} D_{t}^{\alpha} Y=R Y(1-Y)+\frac{K_{2} X Y}{1+I X}-E_{2} Y,
\end{aligned}
$$


with the initial conditions $X(0)=X_{0}$ and $Y(0)=Y_{0}$ and where we define ${ }^{c} D_{t}^{\alpha}$ as the Caputo fractional derivative of order $\alpha$ of a function $f$ by

$$
{ }^{c} D_{t}^{\alpha} f(t)=\frac{1}{\Gamma(n-\alpha)} \int_{c}^{t} \frac{f^{(n)}(s)}{(t-s)^{\alpha+1-n}} \mathrm{~d} s,
$$

and where we have dropped the ${ }^{-}$from $\bar{t}$ for convenience. In (7) the function $f$ is such that $f:[c, \infty) \rightarrow \mathbb{R}, \alpha$ is the order of the fractional derivative, a positive real number such that $n-1<\alpha \leq n$ for $n \in \mathbb{N}, f^{(n)}(s)=\left(\mathrm{d}^{n} / \mathrm{d} s^{n}\right) f(s), \Gamma(\cdot)$ is the Gamma function and $c$ is a constant time at which the state of the system is known and since when memory effects play a role (Podlubny, 1999). In this paper, we will consider the case when $\alpha \in(0,1]$, so that $n=1$, and when $c=0$, i.e. such that memory effects are assumed to include all information since some initial time $t=0$ when the population system was introduced with a known state $X(0)=X_{0}$ and $Y(0)=Y_{0}$. In this case (7) becomes

$$
{ }^{c} D_{t}^{\alpha} f(t)={ }^{0} D_{t}^{\alpha} f(t)=\frac{1}{\Gamma(1-\alpha)} \int_{0}^{t} \frac{f^{(1)}(s)}{(t-s)^{\alpha}} \mathrm{d} s,
$$

which is a weighted integral of $f^{(1)}=\mathrm{d} f / \mathrm{d} t$ with a power-law weighting function that depends on $\alpha$, namely $1 /(t-s)^{\alpha}$. For $\alpha=1$, an application of Cauchy's integral formula shows that the fractional derivative reduces to the integer first derivative, ${ }^{0} D_{t}^{1} f(t)=\mathrm{d} f / \mathrm{d} t$. However, for $0<\alpha<1$, this form of derivative, at time $t$, contains information from all previous times since $t=0$. As the fractional derivative order $\alpha$ decreases towards zero there is increasingly less weight given to $\mathrm{d} f / \mathrm{d} t$ at the present time and more influence from the system at times past.

\section{Equilibrium points}

Since the Caputo derivative of a constant function is zero, the equilibrium points of (5) and (6) can easily be found, and are the same as the integer-order system, namely

$$
\begin{aligned}
& P_{0}=(0,0) \quad \text { the extinction state, } \\
& P_{1}=\left(1-E_{1}, 0\right) \text { the predator-free state, } \\
& P_{2}=\left(0,1-\frac{E_{2}}{R}\right) \text { the prey-free state, } \\
& P_{3 i}=\left(X^{*}, Y^{*}\right) \text { for } i=1,2,3 \text { the coexistence states, }
\end{aligned}
$$

where the coexistence state, i.e. for which $X^{*}>0$ and $Y^{*}>0$, satisfies

$$
Y^{*}=\left(1-\frac{E_{2}}{R}\right)+\frac{K_{2} X^{*}}{R\left(1+I X^{*}\right)}
$$

and

$$
X^{* 3}+C_{2} X^{* 2}+C_{1} X^{*}+C_{0}=0
$$


where

$$
\begin{aligned}
& C_{0}=\frac{1}{I^{2}}\left(E_{1}-1\right)-\frac{K_{1}}{I^{2}}\left(\frac{E_{2}}{R}-1\right), \\
& C_{1}=\frac{2}{I}\left(E_{1}-1\right)-\frac{K_{1}}{I}\left(\frac{E_{2}}{R}-1\right)+\frac{1}{I^{2}}\left(\frac{K_{1} K_{2}}{R}+1\right), \\
& C_{2}=\left(E_{1}-1\right)+\frac{2}{I} .
\end{aligned}
$$

The extinction state, $P_{0}$, where prey and predator populations are zero, the predator-free state, $P_{1}$, and the prey-free state, $P_{2}$, all always exist but if the predator-free and prey-free states are to be physically meaningful we must have $E_{1}<1$ and $E_{2}<R$, respectively. These conditions are equivalent, in dimensional terms, to the limitations that the harvesting rates of the prey and predator are less than the intrinsic growth rates so that $h_{1}<r_{1}$ and $h_{2}<r_{2}$. The coexistence states, $P_{3 i}$, are more complicated and are now discussed in detail.

From (13) we see that, for a physically realistic coexistent state we must, as well as having $X^{*}>0$, have that the harvesting of predators is less than an enhanced growth rate of predators, $E_{2}<R+K_{2} X^{*} /\left(1+I X^{*}\right)$, to ensure that $Y^{*}>0$. For these coexistence states, we therefore see that a higher level of harvesting is possible, compared to the prey-free state, because the non-zero prey population can support the presence of a predator population even with relatively high levels of harvesting. In order to determine the number of real and positive roots of (14) we will consider the discriminant of the equation (see, for instance, discussion of the Cardano formula in van der Waerden, 2003), namely

$$
\Delta=C_{1}^{2} C_{2}^{2}-4 C_{1}^{3}-4 C_{0} C_{2}^{3}-27 C_{0}^{2}+18 C_{0} C_{1} C_{2}
$$

The number of possible coexistence states is determined by the number of positive, real roots of (14) which, using the sign of the discriminant in (18) and Descartes' rule of signs (Anderson, Jackson, \& Sitharam, 1998), lead to the following cases:

one coexistence state $P_{31}$ : if $\Delta<0$ and $E_{1}<M_{0}$,

two coexistence states $P_{31}, P_{32}$ : if $\Delta>0, M_{0}<E_{1}<M_{2}$ and $M_{1}<M_{2}$, two coexistence states $P_{31}, P_{33}$ : if $\Delta>0, M_{0}<E_{1}<M_{1}$ and $M_{2}<M_{1}$, three coexistence states $P_{31}, P_{32}, P_{33}$ : if $\Delta>0$ and $M_{1}<E_{1}<\min \left(M_{0}, M_{2}\right)$,

where

$$
\begin{aligned}
& M_{0}=1+K_{1}\left(\frac{E_{2}}{R}-1\right) \\
& M_{1}=1+\frac{K_{1}}{2}\left(\frac{E_{2}}{R}-1\right)-\frac{1}{2 I}\left(\frac{K_{1} K_{2}}{R}+1\right) \\
& M_{2}=1-\frac{2}{I}
\end{aligned}
$$

are critical values of $E_{1}$ derived from the coefficients $C_{0}, C_{1}, C_{2}$, respectively. 


\section{Stability}

The stability of the equilibria can be determined by considering the boundedness of the solution trajectories and the local stability of each equilibrium point. For boundedness results we refer the reader to Kar et al. (2010), in which it is proved that the system of Equations (3) and (4) are uniformly bounded for trajectories that start in the physically relevant region $X>0, Y>0$. For a fractional derivative system of nonlinear equations, the stability of the various equilibrium points may be investigated using a direct Lyapunov method, to determine the 'Mittag-Lefler stability', as described in Li et al. (2009), or by considering a linearization of the system with the Mittag-Lefler functions acting as eigenfunctions of the system, the equivalent to exponential eigenfunctions in integer-derivative systems, as consider by, for example Alidousti, Khoshsiar Ghaziani, and Bayati Eshkaftaki (2017), Matignon (1996), and Cresson and Szafranska (2017). In this latter approach, determining whether an equilibrium point is stable is undertaken by considering the eigenvalues of the Jacobian matrix, $J(X, Y)$ at the equilibrium point $(X, Y)$. For our system in (5) and (6) the Jacobian matrix is

$$
J(X, Y)=\left[\begin{array}{cc}
\left(1-E_{1}\right)-\frac{K_{1} Y}{(1+I X)^{2}}-2 X & \frac{-K_{1} X}{1+I X} \\
\frac{K_{2} Y}{(1+I X)^{2}} & \left(R-E_{2}\right)+\frac{K_{2} X}{(1+I X)}-2 Y R
\end{array}\right] .
$$

The eigenvalues of the fractional derivative equations, $\lambda$, are related to the eigenvalues of the Jacobian matrix, $\Lambda$, by $\Lambda=\lambda^{\alpha}$ (see, for example, Miller \& Ross, 1993 for a more complete treatment of this approach). Given that the condition for asymptotic stability of the fractional system can be expressed as $\min (|\arg (\lambda)|)>\pi / 2$, i.e. that the real parts of the eigenvalues are negative, the condition for asymptotic stability of the fractional system can therefore be written in terms of the eigenvalues, $\Lambda$, of the integer derivative system thus, $\min (|\arg (\Lambda)|)>\alpha \pi / 2$ (Miller \& Ross, 1993).

This stability condition indicates that, while an equilibrium point may be unstable in the integer-derivative system, i.e. when at least one eigenvalue is such that $\operatorname{Re}(\Lambda)>0$ (or equivalently that $|\arg (\Lambda)|<\pi / 2)$, it may be that the fractional system is stable, provided that $\alpha$ is sufficiently small to allow $|\arg (\Lambda)|>\alpha \pi / 2$. However, for a positive real eigenvalue of the integer-derivative system, so that $\arg (\Lambda)=0$, no non-zero value of $\alpha$ will allow the stability condition to be satisfied. This result means that, since the eigenvalues of the extinction state $P_{0}=(0,0)$, the predator-free state $P_{1}=\left(1-E_{1}, 0\right)$ and the prey-free state $P_{2}=\left(0,1-E_{2} / R\right)$ are all always real, their stability will be unaffected by the fractional derivative order. The stabilities of these three equilibrium points have previously been reported (Kar et al., 2010) but are summarized here for completeness.

The extinction state $P_{0}=(0,0)$ is stable if $E_{1}>1$ and $E_{2}>R$. This result is to be expected and shows that if the harvesting rates are greater than the intrinsic growth rates of the predator and prey species then the extinction state is stable and that if at least one of the harvesting rates is less than the corresponding growth rate then the extinction state is unstable. If the predator-free state $P_{1}=\left(1-E_{1}, 0\right)$ is physical, so that $E_{1}<1$, then the condition for stability is $E_{2}>R+K_{2}\left(1-E_{1}\right) /\left(1+I\left(1-E_{1}\right)\right)$. Therefore, the predatorfree state is stable if it exists and the predator harvesting rate is sufficiently large. If the 
prey-free state $P_{2}=\left(0,1-E_{2} / R\right)$ exists, so that $E_{2}<R$, then the condition for stability is that the prey harvesting rate is sufficiently large, $E_{1}>M_{0}$.

In contrast to the $P_{0}, P_{1}$ and $P_{2}$ states, the stability of the coexistence states $P_{3 i}, i=1$, 2,3 , where the populations of both prey and predator are non-zero, can be affected by the fractional derivative order. The eigenvalues of the Jacobian, (19), corresponding to the coexistence equilibrium points $P_{3 i}, i=1,2,3$, in (12) are the roots of the characteristic equation

$$
\Lambda^{2}+f \Lambda+g=0
$$

where

$$
\begin{aligned}
& f=X^{*}-\frac{K_{1} I Y^{*} X^{*}}{\left(1+I X^{*}\right)^{2}}+R Y^{*}, \\
& g=R Y^{*} X^{*}+\frac{K_{1} Y^{*} X^{*}}{\left(1+I X^{*}\right)^{2}}\left(\frac{K_{2}}{\left(1+I X^{*}\right)}-R I Y^{*}\right),
\end{aligned}
$$

and $\left(X^{*}, Y^{*}\right)$ is one of the three solutions to (13) and (14).

We are then able to derive the following results:

(i) If $f>0$ and $g>0$, then, by the Routh-Hurwitz criterion, (20) has two roots with negative real parts so the equilibrium point is asymptotically stable for all $\alpha \in(0,1]$.

(ii) If $f>0$ and $g<0$, then $g<f^{2} / 4$, so (20) has real roots, and there is one change of sign in the coefficients of (20). Therefore, by the Routh-Hurwitz criterion, (20) has one root with positive real part and the equilibrium point is unstable for all $\alpha \in(0,1]$.

(iii) If $g<f^{2} / 4$ then (20) has real roots and if $f<0$ then there is at least one change of sign in the coefficients of the polynomial in (20) so that, by the Routh-Hurwitz criterion, there is at least one positive real root and the equilibrium point is unstable for all $\alpha \in(0,1]$.

(iv) If $g>f^{2} / 4$ then (20) has two complex conjugate roots with real part $-f / 2$, which is positive since $f<0$. Then, using the stability criterion for the fractional order system, the condition for stability is

$$
\arg \Lambda=\cos ^{-1}\left(-\frac{f}{2 \sqrt{g}}\right)>\frac{\alpha \pi}{2}, \quad \alpha \in(0,1] .
$$

The maximum value of $\alpha$ for which stability is ensured is therefore

$$
\alpha^{*}=\frac{2}{\pi} \cos ^{-1}\left(-\frac{f}{2 \sqrt{g}}\right) .
$$

Thus, if $f<0$ and $g>f^{2} / 4$, the system in (5) and (6) is asymptotically stable if and only if $\alpha \in\left(0, \alpha^{*}\right)$, and when $\alpha \geq \alpha^{*}$ the system is unstable with complex eigenvalues, or equivalently that the system undergoes a Hopf bifurcation at $\left(X^{*}, Y^{*}\right)$ when the fractional order $\alpha$ increases above the critical value $\alpha^{*}$.

We can summarize these results as follows:

(1) If the extinction state $P_{0}$ is a stable equilibrium point, then the predator-free and preyfree states, $P_{1}$ and $P_{2}$, do not both exist. 
(2) The predator-free and prey-free states, $P_{1}$ and $P_{2}$, may co-exist, but they will never both be stable.

(3) If the prey-free state $P_{2}$ is a stable equilibrium point, then the interior equilibrium points $P_{3 i}, i=1,2,3$, do not all exist.

(4) If the extinction state $P_{0}$ is a stable equilibrium point, then the interior equilibrium points $P_{3 i}, i=1,2,3$, do not all exist.

(5) When $E_{1}<1$ and $E_{2}>R+K_{2}\left(1-E_{1}\right) /\left(1+I\left(1-E_{1}\right)\right)$, then $P_{1}$ is a stable point and it is possible for at least one of the coexistence states $P_{3 i}$ to be stable.

(6) When $E_{1}<\min \left(1, M_{0}\right)$ and $E_{2}<R+K_{2}\left(1-E_{1}\right) /\left(1+I\left(1-E_{1}\right)\right)$, then $P_{0}, P_{1}$ and $P_{2}$ are all unstable but it is still possible for at least one of the coexistence states $P_{3 i}$ to be stable.

\section{Numerical simulation}

The results in the last section, specifically point 6 . above, indicate that it may be possible to select parameter values for which there are two stable coexistence equilibrium points (i.e. two of $P_{3 i}, i=1,2,3$ ), and, as indicated in point (iv) above, that the fractional derivative order $\alpha$ can be important in determining the number of stable equilibria. In this section, we investigate the numerical solution of the system in (5) and (6), in just this situation, paying attention to the critical fractional derivative order, $\alpha^{*}$, below the value of which an unstable equilibrium point becomes stable. We will see that, although for $\alpha>\alpha^{*}$ only one equilibrium point is stable, for certain initial states the system can remain close to an unstable equilibrium point. As $\alpha$ reduces the area of initial state space for which the system remains close to this unstable point grows until, when $\alpha<\alpha^{*}$, the unstable point becomes stable. We therefore see that, for $\alpha$ above and below this critical value, the transient behaviour and the observed domains of stability for the equilibria can significantly change as a function of fractional derivative order.

Numerical solutions of (5) and (6) are found using the Adams-Bashforth-Moulton method (Diethelm \& Ford, 2002; Diethelm, Ford, \& Freed, 2002) for parameter values $r_{1}=$ 3 day $^{-1}, r_{2}=0.1$ day $^{-1}, k_{1}=1 \mu \mathrm{L}^{-1}, k_{2}=0.0029 \mu \mathrm{L}^{-1}, m=200 \mathrm{day}^{-1}, a=1001 \mu$ $\mathrm{L}, q=10, h_{1}=0.001 \mathrm{day}^{-1}, h_{2}=0.01 \mathrm{day}^{-1}$ which give the nondimensional parameter values $R=0.0333, K_{1}=0.1935, K_{2}=667.3333, I=1001, E_{1}=0.0003$ and $E_{2}=0.0033$ (Harrison, 1995; Kar et al., 2010; Magal, Cosner, Ruan, \& Casas, 2008), which are typical for systems where the size of predator populations are much larger than prey populations such as in parasitic-predation on host prey (Anderson \& May, 1978), and with a timestep size $h=2^{-8}$ (a value for which the total area of the domains of stability changed by less than 5\% if the timestep $h$ was reduced). This numerical method uses the classical Volterra integral equation form of the initial value problem in Equations (5) and (6), although in the case of fractional derivatives the kernel will have non-integer exponent and the integral has a lower limit of $t=c$ due to the non-local effects of using fractional derivatives. A time-iterative scheme based on this integral form is constructed, leading to an implicit nonlinear equation for the solution at a subsequent time. Using a one-step predictor-corrector method, with the prediction using an explicit evaluation, this equation is solved and time is advanced by one timestep. The quadrature used for evaluation of the integral in the Volterra equation is more involved for a fractional situation since the required approximation is not only for one timestep but for all previous time. However, standard quadrature techniques can be 
used. Full details of this method are given in Diethelm et al. (2002). For a more complete discussion on the numerical analysis of the Adams-Bashforth-Moulton method, the work of Sweilam, Nagy, Assiri, and Ali (2015) and Garrappa (2010) include an error analysis and stability result, respectively, and Leedle (2017) contains a useful summary of many different results, for the standard and higher order Adams-Bashforth-Moulton methods, on uniform and graded time discretizations, including the result that the error is proportional to $h^{1+\alpha}$ for $\alpha<1$.

For these parameter values, the physically relevant equilibrium points are $P_{0}=(0,0)$, $P_{1}=(0.9997,0), P_{2}=(0,0.9009), P_{31}=(0.9956,20.9009), P_{32}=(0.0005,8.0015)$ and $P_{33}=(0.0015,12.9395)$ and $P_{0}, P_{1}, P_{2}, P_{33}$ are all unstable. However, the $P_{31}$ equilibrium point is stable for all values of $\alpha$. For these parameter values, the eigenvalues of the Jacobian matrix at $P_{32}$ are complex conjugates with positive real part, so that for the integer derivative-order system the point $P_{32}$ is unstable. As mentioned above, as the derivative order reduces, the region $|\arg (z)|<\alpha \pi / 2$ eventually expands to include the $P_{32}$ complex conjugate Jacobian eigenvalues, so that for $\alpha \in\left(0, \alpha^{*}\right.$, where $\alpha^{*}=0.88406$, the point $P_{32}$ is stable. This means that for the integer derivative system, i.e. $\alpha=1$, there is only one stable equilibrium point, $P_{31}$, a state in which there are significant levels of prey and predator, but for the fractional derivative system with a fractional derivative order less than the critical value $\alpha<\alpha^{*}$ there exists a further stable equilibrium point, $P_{32}$, a state in which the prey level is almost zero.

We demonstrate this change in stability in Figure 1, which shows the evolution of the system from the same initial condition $X(0)=0.0005, Y(0)=8.0$ but for two fractional derivative-order values, either side of the critical value, for the integer-order system $\alpha=1$ (Figure 1(a)) and $\alpha=0.8$ (Figure 1(b)). In, Figure 1(a) the equilibrium point $P_{32}$ is unstable and the system evolves to the only stable equilibrium point, $P_{31}$. However, for the lower value of $\alpha$, Figure 1(b) shows that, whilst the equilibrium point $P_{31}$ is still stable, for the same initial values of prey and predator populations the system evolves to the, now stable, equilibrium point $P_{32}$.

The fractional derivative order therefore gives the possibility of transitioning from a monostable system to a bistable system. In a classical, integer-order system, the change of stability associated with a pair of complex conjugate eigenvalues moving from the stable to unstable regions of the complex plane (in an integer-order system this would be when eigenvalues move across the imaginary axis, i.e. from $|\arg (z)|<\pi / 2$ to $|\arg (z)|>\pi / 2$ ) is a Hopf bifurcation, with instability occurring through the appearance of a limit cycle around the equilibrium point. However, in a fractional derivative system the dependence of the system on previous times, rather than simply the current time, means that a true limit cycle does not exist and trajectories in the phase plane can pass across a closed cycle or separatrix. A closed cycle can exist, although trajectories do not approach this closed cycle asymptotically, so this is not termed a limit cycle. However, as well as the fractionalderivative-order-induced monostable-bistable transition, changes to such a closed cycle as the fractional derivative order changes mean that significantly changes to the transient behaviour of the population dynamics will occur with changing fractional derivative order.

In Figure 2 we show the phase plane trajectories for prey and predator populations from an initial state of $X(0)=0.0005$ and $Y(0)=8.0$ for six different values of the fractional derivative order above the critical value $\alpha^{*}=0.88406$. In all plots within Figure 2 we see that the equilibrium point $P_{32}$ is unstable, with the trajectory eventually reaching 
(a) fractional derivative order $\alpha=1.0$
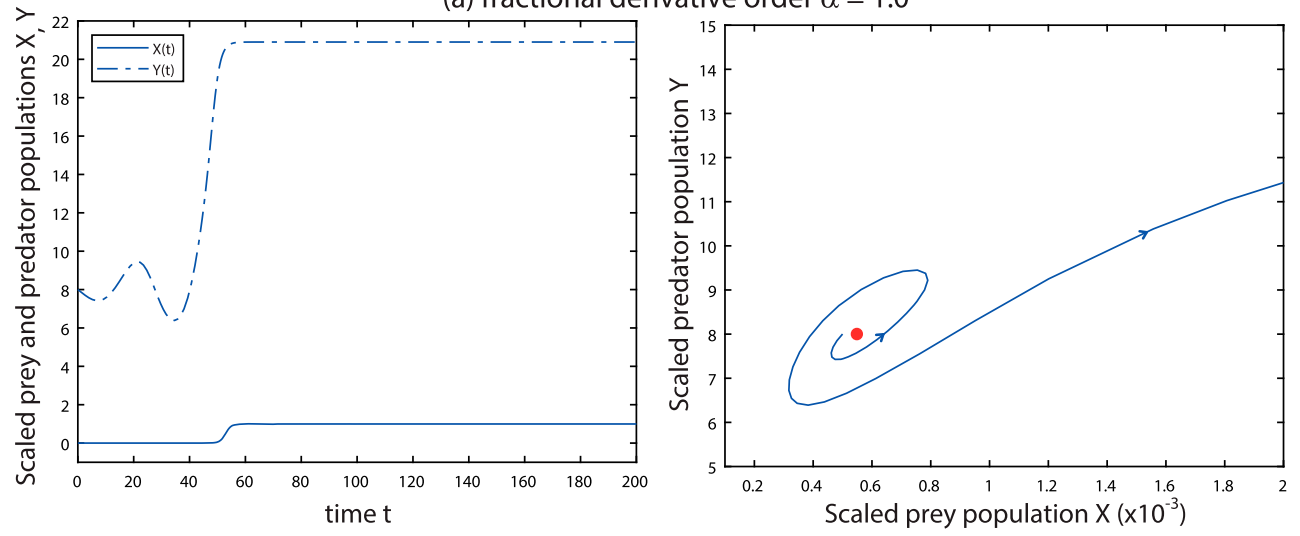

(b) fractional derivative order $\alpha=0.8$
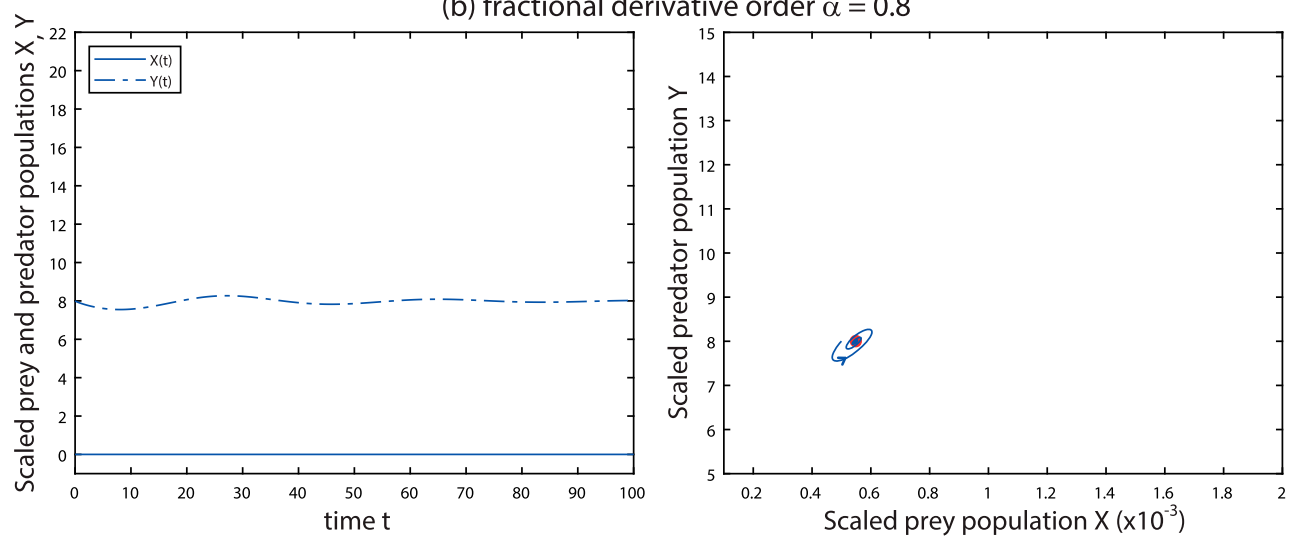

Figure 1. The trajectories from an initial condition $X(0)=0.0005, Y(0)=8.0$ with (a) $\alpha=1>\alpha^{*}$, where the system evolves to the stable equilibrium point $P_{31}=(0.9956,20.9009)$ (not shown in this plot), and (b) $\alpha=0.8<\alpha^{*}$, where the system evolves to the fractional-derivative-stabilized equilibrium point $P_{32}=(0.0005,8.0015)$.

the only stable equilibrium point $P_{31}$. However, as the fractional derivative order changes the transient behaviour, before the system converges to the stable point $P_{32}$, changes. Even though $P_{32}$ is unstable, the Hopf-like bifurcation leads to oscillations in the prey and predator populations. The number of cycles around $P_{32}$ is seen to increase as the fractional derivative order decreases, as shown in Figure 3. When the fractional derivative order has reduced below the critical value $\alpha^{*}=0.88406$ the phase plane trajectory would asymptotically approach $P_{32}$ in an oscillatory fashion, with an infinite number of cycles around the equilibrium point. This approach to an infinite number of cycles is also seen in Figure 3.

The change in transient behaviour can also be seen when we consider the state of the system after a fixed time $t=t_{\text {end }}$. To illustrate this we will now vary the initial state and consider the final state at $t=t_{\text {end }}=500$ (equivalent to a dimensional $t=167$ days), labelling each initial point depending on whether the final state is closer to $P_{31}$ or $P_{32}$. Figure 4 shows the results of this process of labelling the initial states depending on whether the final state is closer to $P_{31}$ (coloured grey (green online)) or $P_{32}$ (coloured white). For values of the initial state $X_{0}$ greater than 0.1 the system final state, at $t_{\text {end }}$, is always closer to 

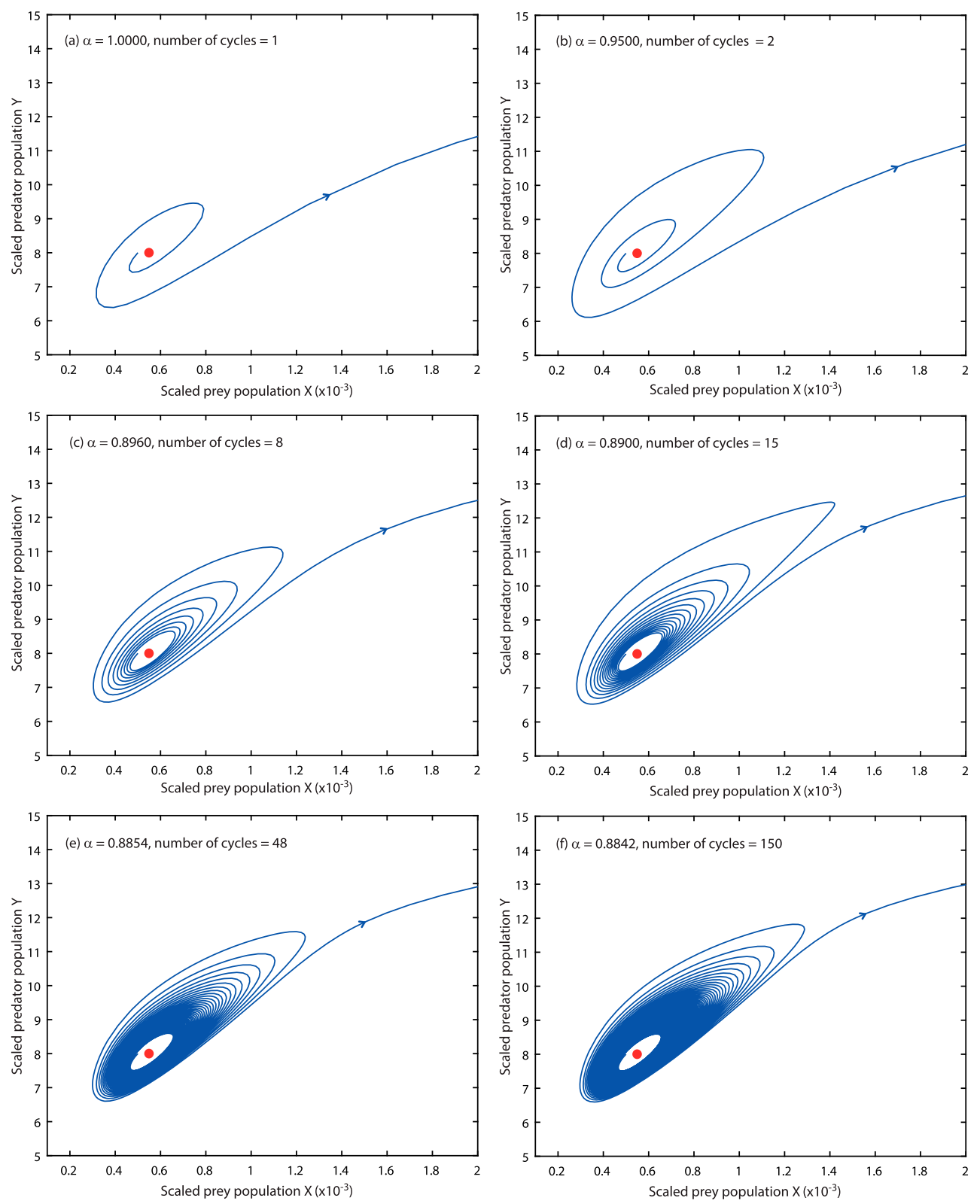

Figure 2. Phase plane trajectories for the predator-prey populations for the initial state $X(0)=0.0005$, $Y(0)=8.0$ but for various values of the fractional derivative order $\alpha$. The dot marks the equilibrium point $P_{32}=(0.0005,8.0015)$. As $\alpha$ decreases (from (a) to (f)) the number of cycles around $P_{32}$ increases.

$P_{31}$ and would therefore be coloured grey (green online). For the value of fractional derivative order used for Figure 4, $\alpha=0.75<\alpha^{*}$, i.e. below the critical value, we see that both $P_{31}$ and $P_{32}$ are stable, although the domain of attraction of $P_{31}$ is much larger than $P_{32}$. Figure 5 then shows an enlarged version of Figure 4, indicating how the domain of attraction of $P_{32}$ changes as the fractional derivative order changes. From Figure $5(d-f)$ we see 


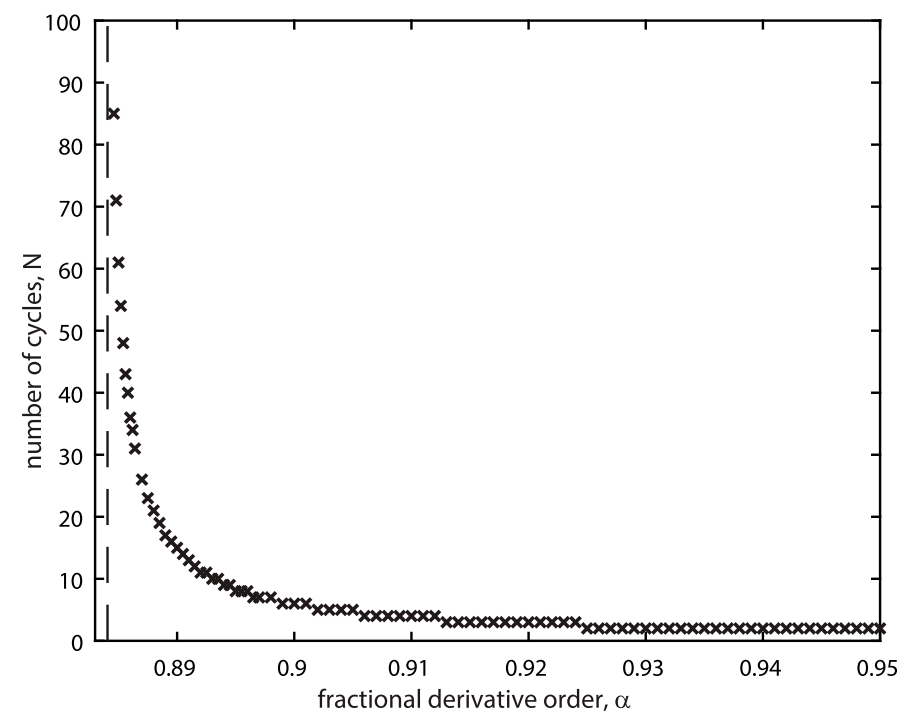

Figure 3. The number of cycles around the equilibrium point $P_{32}$ as the fractional derivative order $\alpha$ varies as was illustrated in Figure 2.

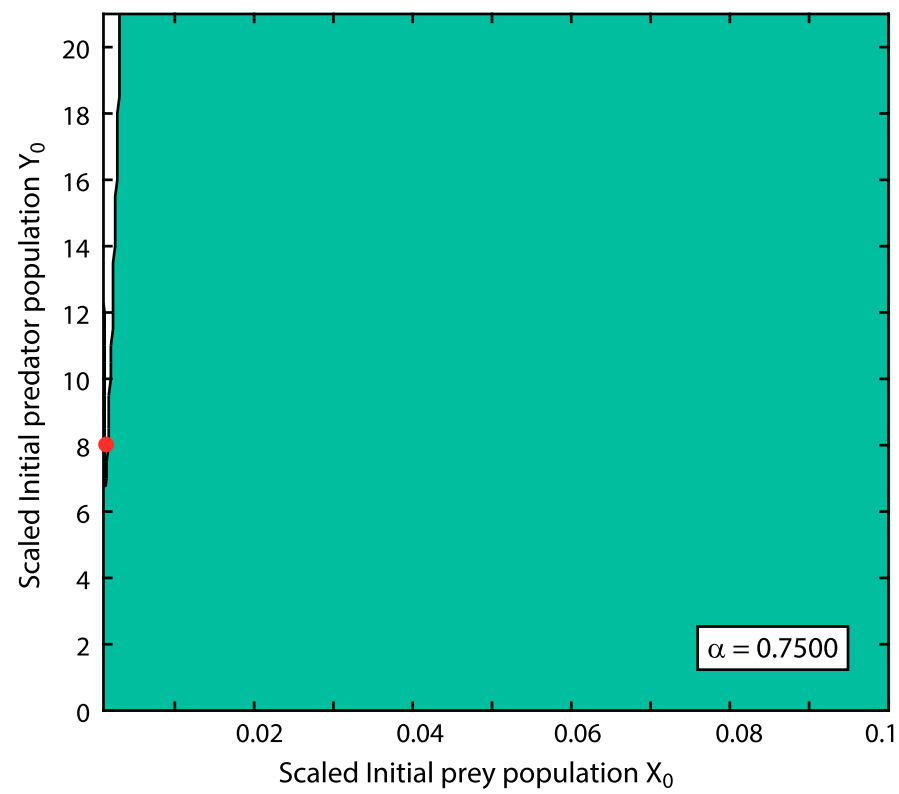

Figure 4. Observed domains of attraction for $\alpha=0.75<\alpha^{*}$. If the initial states $X_{0}, Y_{0}$ are chosen from the grey (green online) region the system will be closer to the equilibrium point $P_{31}$ at $t=t_{\text {end }}$, and for initial states within the white region the system be closer to the equilibrium point $P_{32}$ at $t=t_{\text {end }}$.

that for fractional derivative orders less than the critical value, for which both $P_{31}$ and $P_{32}$ are stable, we have a region of the space of initial states where the system is closer to $P_{31}$ when $t=t_{\text {end }}$ and a region where the system is closer to $P_{32}$. When the fractional derivative order is greater than the critical value Figure $5(a-c)$ we might expect, since the equilibrium 

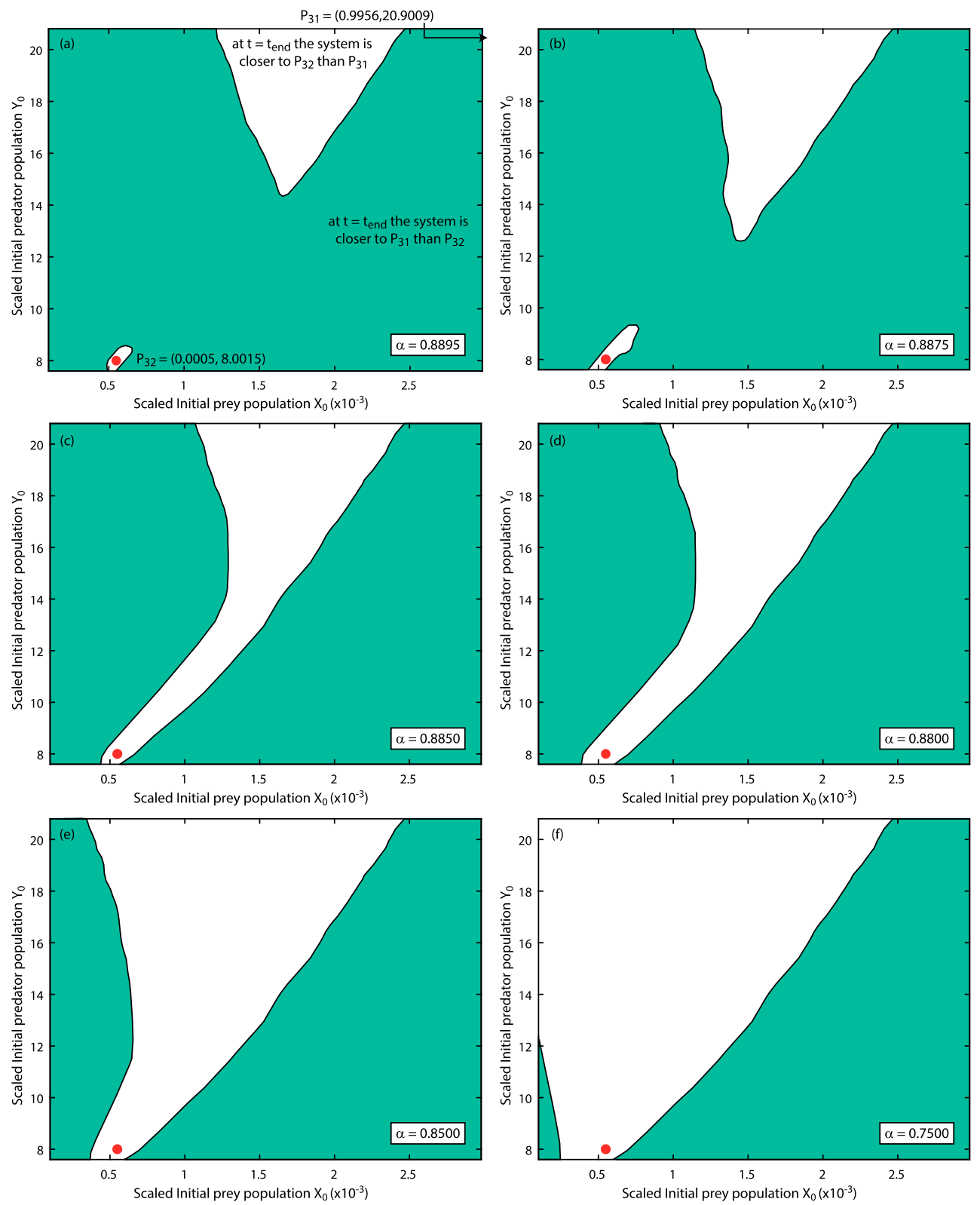

Figure 5. Observed domains of attraction for various values of the fractional derivative order $\alpha$. If the initial states $X_{0}, Y_{0}$ are chosen from the grey (green online) region the system will be closer to the equilibrium point $P_{31}$ at $t=t_{\text {end }}$, and for initial states within the white region the system be closer to the equilibrium point $P_{32}$ at $t=t_{\text {end }}$.

point $P_{32}$ is now unstable, that the white region in these plots would collapse. However, since these regions are labelled after a fixed time $t=t_{\text {end }}$, and because of the Hopf-like bifurcation that leads to oscillatory behaviour around $P_{32}$, we see that, for a range of initial conditions, the system remains close to $P_{32}$. It is interesting to note that for relatively large values of the fractional derivative order, i.e. in Figure $5(a, b)$ there are two disconnected 
white regions. Further investigation of the fine structure of the boundaries of these disconnected regions is necessary, although this will also depend on the value of $t_{\text {end }}$ that is used, and may for instance discover fractal domains of attraction such as those found in systems of delay-differential equations (Taylor \& Campbell, 2007). A key observation from Figure 5 is that, both for fractional derivatives below and above the critical value $\alpha^{*}$ the observed domain of attraction changes significantly as $\alpha$ changes. In a real-life ecological setting the value of $\alpha$, i.e. the memory characteristics of species, may be fixed, but Figure 5 shows that the long-term state of the system may be unpredictable given the complicated nature of the observed domain of attraction. However, we can speculate that the memory function of species could vary, leading to the changes in the domains of attraction shown in Figure 5. Such changes could occur in time or age class, as memory is often a function of experience or age (Gordon, 1983), but possibly also with environmental factors (Klosin, Casas, Hidalgo-Carcedo, Vavouri, \& Lehner, 2017). Of course, without a satisfactory link between a model of memory function and the fractional derivative model of population dynamics this is only speculation, but an understanding of the changes in the domains of attraction as a function of $\alpha$ may prove useful for a comparison between experimental or field trial work and theoretical models.

\section{Conclusions}

In this paper, that statics and dynamics of a fractional-order predator-prey model have been investigated, where the fractional derivative order is used to model a form of memory effect in the predator and prey populations. The model of memory, through the use of fractional derivatives and the single parameter $\alpha$ is phenomenological, but has proved useful in previous situations where theory and experiments have been compared (Bolton et al., 2014; Caputo, 2018; Caputo \& Cametti, 2008, 2009; Caputo \& Carcione, 2013; Du et al., 2013). For the system under consideration, conditions of stability and bifurcation have been obtained and, importantly, it was found that through the variation of the fractional order $\alpha$ it is possible to transition from a monostable system to a bistable system. In the bistable system the two stable equilibrium points $\left(P_{31}\right.$ and $\left.P_{31}\right)$ have very different levels of predator and prey populations - for the parameters used here we find prey/predator ratios of $X / Y=0.04760$ and $X / Y=0.00006$ for the two bistable states. The presence of increased long-term memory (corresponding to a reduction in $\alpha$ ) in the predator and prey has therefore opened up the possibility of very different stable states and, at the boundary of the domain of attraction, a sensitivity to small variations in system parameters, such as birth, death or harvesting rates, can lead to a drastic change in the prey/predator ratio. Even when the system has only one stable state, the observed domain of attraction (the domains for which the system will remain close to either equilibrium point after a fixed time) can be memory dependent. Here we have concentrated on a particular model, with the same parameters as used in Kar et al. (2010). For these parameter values, the domain of attraction for $P_{32}$ is much smaller than that of $P_{31}$ and so may not be readily observable in real life. However, we would suggest that the main effects, on stability and domains of attraction, of altering the fractional derivative order would be present for any similar system where there are more than one stable state possible. A more complete investigation of the parameter space, an investigation of the fine structure of the boundary of the domain of attraction, and further extensions to this model, including an incommensurate fractional 
system, for which the predator and prey populations have different fractional derivative orders, and their consequences and conclusions for real-life systems would be extremely interesting to attempt in the future.

\section{Disclosure statement}

No potential conflict of interest was reported by the authors.

\section{Funding}

One of the authors (N.J.M.) was partially supported by the United Kingdom Medical Research Council (MRC) via grant no. G0902331.

\section{ORCID}

Z. M. Alqahtani (i) http://orcid.org/0000-0002-6726-6597

M. El-shahed (D) http://orcid.org/0000-0001-9508-3192

N. J. Mottram (iD http://orcid.org/0000-0002-7265-0059

\section{References}

Alidousti, J., Khoshsiar Ghaziani, R., \& Bayati Eshkaftaki, A. (2017). Stability analysis of nonlinear fractional differential order systems with Caputo and Riemann-Liouville derivatives. Turkish Journal of Mathematics, 41, 1260-1278.

Anderson, B., Jackson, J., \& Sitharam, M. (1998). Descartes' rule of signs revisited. The American Mathematical Monthly, 105, 447-451.

Anderson, R. M., \& May, R. M. (1978). Regulation and stability of host-parasite population interactions: I. Regulatory processes. Journal of Animal Ecology, 47, 219-247.

Arshad, S., Baleanu, D., Huang, J., Tang, Y., \& Al Qurashi, M. M. (2016). Dynamical analysis of fractional order model of immunogenic tumors. Advances in Mechanical Engineering, 8, 1-13.

Bolton, L., Cloot, A. H., Schoombie, S. W., \& Slabbert, J. P. (2014). A proposed fractional-order Gompertz model and its application to tumour growth data. Mathematical Medicine and Biology: A Journal of the IMA, 32, 187-207.

Caputo, M. (1967). Linear models of dissipation whose $Q$ is almost frequency independent. Geophysical Journal International, 13, 529-539.

Caputo, M. (2016). The unknown set of memory constitutive equations of plastic media. Progress in Fractional Differentiation and Applications, 2, 77-83.

Caputo, M. (2018). Memory in constitutive equations and auxetic media. Rendiconti Lincei. Scienze Fisiche e Naturali, 29, S7-S9.

Caputo, M., \& Cametti, C. (2008). Diffusion with memory in two cases of biological interest. Journal of Theoretical Biology, 254, 697-703.

Caputo, M., \& Cametti, C. (2009). The memory formalism in the diffusion of drugs through skin membrane. Journal of Physics D: Applied Physics, 42, 125505.

Caputo, M., \& Carcione, J. M. (2013). A memory model of sedimentation in water reservoirs. Journal of Hydrology, 476, 426-432.

Caputo, M., \& Mainardi, F. (1971). A new dissipation model based on memory mechanism. Pure and Applied Geophysics, 91, 134-147.

Chattopadhyay, J., Sarkar, R. R., \& Ghosal, G. (2002). Removal of infected prey prevent limit cycle oscillations in an infected prey-predator system a mathematical study. Ecological Modelling, 156, 113-121.

Cresson, J., \& Szafranska, A. (2017). Discrete and continuous fractional persistence problems the positivity property and applications. Communications in Nonlinear Science and Numerical Simulation, 44, 424-448. 
Dai, G., \& Tang, M. (1998). Coexistence region and global dynamics of a harvested predator-prey system. SIAM Journal on Applied Mathematics, 58, 193-210.

Dawes, J. H. P., \& Souza, M. O. (2013). A derivation of Holling's type I, II and III functional responses in predator-prey systems. Journal of Theoretical Biology, 327, 11-22.

Diethelm, K., \& Ford, N. J. (2002). Analysis of fractional differential equations. Journal of Mathematical Analysis and Applications, 265, 229-248.

Diethelm, K., Ford, N. J., \& Freed, A. D. (2002). A predictor-corrector approach for the numerical solution of fractional differential equations. Nonlinear Dynamics, 29, 3-22.

Du, M., Wang, Z., \& Hu, H. (2013). Measuring memory with the order of fractional derivative. Scientific Reports, 3, 3431.

El-Shahed, M., \& Alsaedi, A. (2011). The fractional SIRC model and influenza A. Mathematical Problems in Engineering, 2011, 480378.

Fenton, A., \& Perkins, S. E. (2010). Applying predator-prey theory to modelling immune-mediated, within-host interspecific parasite interactions. Parasitology, 137, 1027-1038.

Forde, J. E. (2005). Delay differential equation models in mathematical biology (PhD thesis). University of Michigan.

Freedman, H. I. (1980). Deterministic mathematical models in population ecology. New York, NY: Marcel Dekker.

Freedman, H. I., \& Rao, V. S. H. (1986). Stability criteria for a system involving two time delays. SIAM Journal on Applied Mathematics, 46, 552-560.

Freedman, H. I., \& Waltman, P. (1984). Persistence in models of three interacting predator-prey populations. Mathematical Biosciences, 68, 213-231.

Garrappa, R. (2010). On linear stability of predictor-corrector algorithms for fractional differential equations. International Journal of Computer Mathematics, 87, 2281-2290.

Gordon, W. C. (1983). The malleability of memory in animals. Advances in Psychology, 13, 399-426.

Harrison, G. W. (1995). Comparing predator-prey models to Luckinbill's experiment with didinium and paramecium. Ecology, 76, 357-374.

Heymans, N., \& Podlubny, I. (2006). Physical interpretation of initial conditions for fractional differential equations with Riemann-Liouville fractional derivatives. Rheologica Acta, 45, 765-771.

Kar, T. K., Chakraborty, K., \& Pahari, U. K. (2010). A prey-predator model with alternative prey. Canadian Applied Mathematics Quarterly, 18, 137-168.

Kilbas, A. A., Srivastava, H. M., \& Trujillo, J. J. (2006). Theory and applications of fractional differential equations. Amsterdam: Elsevier Science.

Klosin, A., Casas, E., Hidalgo-Carcedo, C., Vavouri, T., \& Lehner, B. (2017). Transgenerational transmission of environmental information in C. Elegans. Science, 356, 320-323.

Kuang, Y. (1993). Delay differential equations: With applications in population dynamics. Boston, MA: Academic Press.

Leedle, N. (2017). A new predictor-corrector method for solving nonlinear fractional differential equations with graded meshes ( $\mathrm{PhD}$ thesis). University of Chester.

Li, Y., Chen, Y., \& Podlubny, I. (2009). Mittag Leffler stability of fractional order nonlinear dynamic systems. Automatica, 45, 1965-1969.

Magal, C., Cosner, C., Ruan, S., \& Casas, J. (2008). Control of invasive hosts by generalist parasitoids. Mathematical Medicine and Biology, 25, 1-20.

Matignon, D. (1996). Stability results for fractional differential equations with applications to control processing. Computational Engineering in Systems and Applications, 2, 963-968.

Miller, K. S., \& Ross, B. (1993). An introduction to the fractional calculus and fractional differential equations. New York, NY: Wiley.

Milton, J. G. (2015). Time delays and the control of biological systems: An overview. International Federation of Automatic Control, 48, 87-92.

Murray, J. D. (2004). Mathematical biology. Interdisciplinary applied mathematics: 17. Berlin: Springer Verlag.

Oldham, K. B., \& Spanier, J. (1974). The fractional calculus theory and applications of differentiation and integration to arbitrary order. San Diego, CA: Academic Press. 
Pinto, C. M., \& Carvalho, A. R. (2017). The HIV/TB co-infection severity in the presence of TB multi-drug resistant strains. Ecological Complexity, 32, 1-20.

Podlubny, I. (1999). Fractional differential equations. San Diego, CA: Academic Press.

Pooseh, S., Rodrigues, H. S., \& Torres, D. F. M. (2011). Fractional derivatives in dengue epidemics. AIP Conference Proceedings, 1389, 739-742.

Rahman, M. S., \& Chakravarty, S. (2013). A predator-prey model with disease in prey. Nonlinear Analysis: Modelling and Control, 18, 191-209.

Richard, H. (2014). Fractional calculus: An introduction for physicists. Hackensack: World Scientific.

Rihan, F. A. (2013). Numerical modeling of fractional-order biological systems. Abstract and Applied Analysis, 2013, 816803.

Srinivasu, P. D. N., Ismail, S., \& Naidu, C. R. (2001). Global dynamics and controllability of a harvested prey-predator system. Journal of Biological Systems, 9, 67-79.

Sweilam, N. H., Nagy, A. M., Assiri, T. A., \& Ali, N. Y. (2015). Numerical simulations for variableorder fractional nonlinear delay differential equations. Journal of Fractional Calculus and Applications, 6, 71-82.

Taylor, S. R., \& Campbell, S. A. (2007). Approximating chaotic saddles for delay differential equations. Physical Review E, 75, 046215.

van der Waerden, B. L. (2003). Algebra. New York, NY: Springer-Verlag.

Zhang, F., Chen, G., Li, C., \& Kurths, J. (2013). Chaos synchronization in fractional differential systems. Philosophical Transactions of the Royal Society A, 371, 2012.0155. 